\title{
Influencia del tratamiento térmico (tostado) en semillas de chía sobre su calidad nutrimental
}

\section{Influence of heat treatment (roasting) in chia seeds on its nutritional quality}

DOI: $10.46932 / \mathrm{sfjdv2n5-083}$

Received in: Oct 1st, 2021

Accepted in: Dec 30th, 2021

\section{Enrique martínez-manrique}

doctor en ciencias

laboratorio de bioquímica y fisiología de granos (labifigras), facultad de estudios superiores cuautitlán, universidad nacional autónoma de méxico

km 2.5 carretera cuautitlán-teoloyucan, cuautitlán izcalli, méxico, cp 54700

E-mail: tallerdecereales.fesc@yahoo.com.mx

\section{verónica jiménez-vera}

ingeniera en alimentos

laboratorio de bioquímica y fisiología de granos (labifigras), facultad de estudios superiores cuautitlán, universidad nacional autónoma de méxico

km 2.5 carretera cuautitlán-teoloyucan, cuautitlán izcalli, méxico, cp 54700

E-mail: tallerdecereales.fesc@yahoo.com.mx

\section{RESUMEN}

La chía fue una semilla muy consumida por los Mayas y Aztecas; ellos la utilizaban tostada y molida para la preparación de bebidas o para conservarla. Por eso, hoy en día el tostado de la semilla puede ser una opción para mejorar su molienda y aumentar su consumo, pues este grano tiene una alta calidad nutrimental, pero las altas temperaturas podrían afectar esta cualidad. Por esto, se planteó estudiar si la chía disminuye su calidad nutrimental al ser sometida a un tratamiento térmico (tostado); realizando el tostado de la semilla a diferentes tiempos y evaluando su composición química proximal, digestibilidad in vitro, triptófano y factores antinutrimentales. Los resultados mostraron que a mayor tiempo de tostado disminuye su contenido de proteína, grasa y fibra; aumenta su digestibilidad y disminuye el triptófano y sus factores antinutrimentales.

Palabras claves: Chía, semillas, tostado, calidad nutrimental.

\begin{abstract}
Chia was a seed widely consumed by the Mayans and Aztecs; they used it toasted and ground for the preparation of drinks or to preserve it. For this reason, today the roasting of the seed can be an option to improve its grinding and increase its consumption, since this grain has a high nutritional quality, but high temperatures could affect this quality. For this reason, it was proposed to study whether chia decreases its nutritional quality when subjected to heat treatment (roasting); roasting the seed at different times and evaluating its proximal chemical composition, in vitro digestibility, tryptophan and antinutrient factors. The results showed that the longer the roasting time decreases its protein, fat and fiber content; increases its digestibility and decreases tryptophan and its anti-nutritional factors.
\end{abstract}

Keywords: Chia, seeds, roasted, nutritional quality. 


\section{INTRODUCCIÓN}

La chía (Salvia hispanica L.) es una especie que pertenece a la familia de la Labiatae, donde también se encuentran algunas plantas aromáticas como la menta, el tomillo, el romero y el orégano. Es una semilla nativa del sur de México. El uso de la semilla se remonta a la época de los Mayas y los Aztecas, quienes empleaban la semilla como alimento, medicina, ofrenda a los dioses y materia prima para producir un aceite que era empleado como base en pinturas decorativas y ungüentos cosméticos (Fernández, 2010).

Con la llegada de los españoles, las tradiciones de los nativos fueron suprimidas y la mayor parte de su agricultura intensiva y su sistema de comercialización destruidos. Muchos cultivos que habían tenido preponderancia en las dietas precolombinas fueron prohibidos por los españoles debido a su estrecha asociación con los cultos religiosos, siendo reemplazados por otros cultivos (trigo, cebada, arroz) demandados por los conquistadores (Guiotto, 2014).

En la actualidad la semilla de chía es considerada un alimento con alto valor nutritivo. La ciencia actual explica porque las antiguas civilizaciones consideraban a la chía un componente básico en su dieta. La chía es una buena fuente de omega 3 y de antioxidantes. De esta forma, los antioxidantes de la semilla de chía eliminan la necesidad de utilizar antioxidantes artificiales, dándole una enorme ventaja sobre otras fuentes de ácidos grasos omega 3, y permiten almacenarlas por años, sin que se deteriore ni enrancie el sabor o el olor (Guiotto, 2014). Todo esto ha hecho que la chía sea utilizada en diferentes procesos, con tratamientos previos como son: hidratadas en agua fría, hidratadas en agua caliente, tostadas y molidas (harina) (Silva, 2015)

La harina tostada es el subproductos de la chía más utilizado, antiguamente era usado para la elaboración de una popular bebida nutritiva denominada "Chía fresca” (agua, limón, chía) costumbre que, con variantes, persiste hoy en Centroamérica y se denomina "Agua Fresca de Chía” (Ayerza y Coates, 2006). Los indios (así los llamaban), preparaban con la semilla de chía, tostada en una patena o en un comalli, como ellos le llamaban, una especie de harina que se guardaba para el uso aún después de algunos años. Luego que estaba hecha polvo o molida, le mezclaban agua, y la agitaban hasta que se obtenía de una densidad agradable al paladar (Urbina, 1987).

Por otro lado, se sabe que el tratamiento térmico puede mejorar la calidad nutrimental de algunos granos como el amaranto (Jiménez-Vera et al; 2014) o disminuirla como en el caso del frijol (MartínezManrique et al; 2005), por lo que, aunque el tostado de la chía puede tener ciertas ventajas, como se ha mencionado antes, no se sabe el efecto del tratamiento térmico sobre su calidad nutrimental. Por eso, se ha planteado en este trabajo, evaluar el efecto del tostado sobre la calidad nutrimental del grano de chía 


\section{METODOLOGÍA}

Para este trabajo se utilizó chía del estado de Puebla, México, cosecha 2015. La cual fue sometida a un tratamiento térmico $\left(170^{\circ} \mathrm{C}\right)$ por diferentes tiempos $(0,5,7.5,10,12.5$ y 15 minutos $)$. Las muestras tostadas se molieron con un molino de cuchillas con una malla \#40 USA serie Tyler. La harina se sometió a un Análisis Químico Proximal (AOAC, 2005), determinándose Humedad (925.09B), Grasa (920.35), Proteínas (991.20), Cenizas (923.03), Fibra (985.29) y Carbohidratos por diferencia. También se evaluó su Digestibilidad (Hsu et al, 1977) contenido de Triptófano (Rama et al, 1974) y de factores antinutrimentales Taninos (ISO 9648, 1988,) y Ácido Fítico (Haug et al., 1983).

\section{RESULTADOS Y DISCUSIÓN}

En la tabla 1 podemos observar que la humedad disminuye, a partir de los 5 minutos de tratamiento térmico (tostado), hasta casi cero, como era de esperarse. Las proteínas disminuyen ligeramente y las cenizas no se modifican. La fibra disminuyó a partir de los 5 minutos de tostado pero se mantuvo sin diferencia estadísticamente significativa $(\mathrm{P}<0.05)$. Sin embargo, el cambio más drástico se observó en la grasa, pues disminuyó casi en un 70\% a partir de los 5 minutos de tostado, eso podría deberse a que dicho tratamiento reblandece el mesocarpio y daña los cuerpos lipídicos que se encuentran en los cotiledones del embrión perdiéndose el aceite (Di Sapio et al, 2012), porque incluso los granos tostados se sienten grasosos.

\begin{tabular}{|c|c|c|c|c|c|c|}
\hline $\begin{array}{l}\text { TIEMPO } \\
\text { (minutos) }\end{array}$ & $\begin{array}{c}\text { HUMEDAD } \\
\% \\
\end{array}$ & $\begin{array}{c}\text { PROTEÍNA } \\
\%\end{array}$ & $\begin{array}{c}\text { CENIZA } \\
\% \\
\end{array}$ & $\begin{array}{c}\text { GRASA } \\
\% \\
\end{array}$ & $\begin{array}{c}\text { FIBRA } \\
\%\end{array}$ & $\begin{array}{c}\text { CHO'S }^{\prime} \\
\%\end{array}$ \\
\hline$\overline{0}$ & $7.83 \pm 0.07^{\mathrm{a}^{*}}$ & $21.54 \pm 0.47^{\mathrm{a}}$ & $3.55 \pm 0.09^{a}$ & $32.14 \pm 0.5^{a}$ & $32.85 \pm 0.83^{a}$ & $2.09^{a}$ \\
\hline 5 & $0.66 \pm 0.03^{b}$ & $19.91 \pm 0.57^{\mathrm{ab}}$ & $3.83 \pm 0.05^{\mathrm{ab}}$ & $8.74 \pm 0.19^{b}$ & $27.03 \pm 0.1^{b}$ & $40.03^{b}$ \\
\hline 7.5 & $0.48 \pm 0.48^{c}$ & $19.21 \pm 0.04^{\mathrm{ab}}$ & $4.25 \pm 0.12^{b}$ & $9.04 \pm 0.13^{\mathrm{b}}$ & $30.4 \pm 0.7^{\mathrm{ab}}$ & $36.62^{b}$ \\
\hline 10 & $0.23 \pm 0.01^{\mathrm{d}}$ & $18.31 \pm 0.03^{b}$ & $3.79 \pm 0.12^{\mathrm{ab}}$ & $10-52 \pm 0.43^{c}$ & $28.38 \pm 0.51^{b}$ & $38.77^{\mathrm{b}}$ \\
\hline 12.5 & $0.24 \pm 0.01^{\mathrm{d}}$ & $18.19 \pm 0.04^{b}$ & $3.79 \pm 0.01^{\mathrm{ab}}$ & $10.78 \pm 0.08^{c}$ & $26.61 \pm 0.5^{b}$ & $40.39^{b}$ \\
\hline 15 & $0.26 \pm 0.02^{\mathrm{d}}$ & $17.4 \pm 0.03^{b}$ & $3.78 \pm 0.01^{\mathrm{ab}}$ & $10.83 \pm 0.08^{c}$ & $29.97 \pm 0.15^{b}$ & $37.76^{\mathrm{b}}$ \\
\hline
\end{tabular}

*Diferentes letras en una columna indican diferencia estadísticamente significativa $\mathrm{P} \leq 0.05$

La digestibilidad (tabla 2) aumentó en relación directa al tiempo de tratamiento térmico, pero a los 15 minutos disminuyó, esto podría ser porque el tostado favorece la hidrólisis de proteína y mejora su digestibilidad, pero a los 15 minutos este tratamiento es muy drástico y dañó las proteína presentes en la chía, pues también disminuyó la concentración de triptófano en este tiempo de tostado (tabla 2), considerando que la molécula de triptófano es termosensible (Cortés, 2011), posiblemente el tratamiento térmico la está dañando. 
Tabla 2. Porcentaje de Digestibilidad in vitro y contenido de Triptófano en la chía a diferentes tiempos de tostada

\begin{tabular}{ccc}
\hline $\begin{array}{c}\text { TIEMPO } \\
\text { (minutos) }\end{array}$ & $\begin{array}{c}\text { DIGESTIBILIDAD } \\
\text { in vitro }\end{array}$ & $\begin{array}{c}\text { TRIPTÓFANO } \\
\text { g aa/g proteína }\end{array}$ \\
\hline $\mathbf{0}$ & $80.28 \pm 0.4^{\mathrm{a}^{*}}$ & $1.81 \pm 0.07^{\mathrm{a}}$ \\
$\mathbf{5}$ & $81.78 \pm 1.02^{\mathrm{b}}$ & $1.23 \pm 0.07^{\mathrm{b}}$ \\
$\mathbf{7 . 5}$ & $83.52 \pm 0.45^{\mathrm{b}}$ & $0.98 \pm 0.07^{\mathrm{c}}$ \\
$\mathbf{1 0}$ & $84.57 \pm 0.56^{\mathrm{b}}$ & $0.81 \pm 0.01^{\mathrm{d}}$ \\
$\mathbf{1 2 . 5}$ & $84.27 \pm 0.46^{\mathrm{b}}$ & $0.60 \pm 0.03^{\mathrm{e}}$ \\
$\mathbf{1 5}$ & $82.73 \pm 0.3^{\mathrm{b}}$ & $0.52 \pm 0.01^{\mathrm{e}}$ \\
\hline
\end{tabular}

*Diferentes letras en una columna indican diferencia estadísticamente significativa $\mathrm{P} \leq 0.05$

Las pruebas de factores antinutrimentales (tabla 3) indican que existe un efecto positivo del tostado sobre estos compuestos, pues en los dos casos disminuyó su contenido en relación directa al tiempo de tostado, aunque en los taninos hubo un mayor efecto pues disminuyeron aproximadamente en un $40 \%$ con respecto al tiempo cero de tostado, mientras que el ácido fítico solo disminuyó un 30\%.

Tabla 3. Contenido de Taninos y Ácido Fítico presentes en la chía a diferentes tiempos de tostada.

\begin{tabular}{ccc}
\hline $\begin{array}{c}\text { TIEMPO } \\
\text { (minutos) }\end{array}$ & $\begin{array}{c}\text { TANINOS } \\
\text { \% }\end{array}$ & $\begin{array}{c}\text { ACIDO FITICO } \\
\text { \% }\end{array}$ \\
\hline $\mathbf{0}$ & $0.4 \pm 0.03^{\mathrm{a}^{*}}$ & $1.15 \pm 0.04^{\mathrm{a}}$ \\
$\mathbf{5}$ & $0.34 \pm 0.02^{\mathrm{b}}$ & $0.94 \pm 0.06^{\mathrm{b}}$ \\
$\mathbf{7 . 5}$ & $0.33 \pm 0.01^{\mathrm{b}}$ & $0.91 \pm 0.12^{\mathrm{b}}$ \\
$\mathbf{1 0}$ & $0.3 \pm 0.02^{\mathrm{b}}$ & $0.89 \pm 0.08^{\mathrm{b}}$ \\
$\mathbf{1 2 . 5}$ & $0.25 \pm 0.01^{\mathrm{c}}$ & $0.82 \pm 0.09^{\mathrm{b}}$ \\
$\mathbf{1 5}$ & $0.23 \pm 0.0007^{\mathrm{c}}$ & $0.85 \pm 0.07^{\mathrm{b}}$ \\
\hline
\end{tabular}

*Diferentes letras en una columna indican diferencia estadísticamente significativa $\mathrm{P} \leq 0.05$

\section{CONCLUSIONES}

Se puede concluir que el tratamiento térmico (tostado) de los granos de chía afectó negativamente su contenido de grasa y triptófano; pero también tuvo un efecto positivo pues mejoró su digestibilidad y disminuyó su contenido de compuestos antinutrimentales.

\section{AGRADECIMIENTOS}

Trabajo realizado con apoyo del proyecto PIAPI-2028 de la FES-Cuautitlán, UNAM. 


\section{REFERENCIAS}

1. A.O.A.C. (2005). Official Methods of Analysis. 18th ed. Association of Official Analytical ChemistsInternational Gaithersburg, Maryland, USA.

2. Ayerza R. y Coates W. (2006). Chía: Redescubriendo un olvidado alimento Azteca. University of Arizona Press.

3. Córtes Avila Yenifer D. (2011). Desarrollo de un pan complementado con harina de amaranto (Amaranthus hypochondriacus) con alta calidad nutrimental. Tesis Licenciatura. FES-Cuautitlán, UNAM.

4. Di Sapio, O., Bueno, M., Busilacci, H., Quiroga, M., Severin, C. (2012). Caracterización Morfo anatómica de Hoja, Tallo, Fruto y Semilla de Salvia hispanica L. (Lamiaceae). Boletín Latinoamericano y del Caribe de Plantas Medicinales y Aromáticas, 11(3):249-268.

5. Jiménez-Vera, J., Argueta, K., Martínez-Manrique, E. (2014). Efecto del tratamiento térmico del amaranto deteriorado sobre su calidad nutrimental. Memorias $49^{\circ}$ Congreso Mexicano de Química y $33^{\circ}$ Congreso Nacional de Educación Química. 17 al 21 de septiembre, Mérida, Yucatán, México.

6. Guiotto Estefania N. (2014). Aplicación de subproductos de chía (Salvia hispanica) y girasol (Helianthus annuus L.) Tesis Doctoral. F. Ciencias Exactas. U. Nacional de La Plata. Argentina.

7. Haug, W., Lantzsch, H., (1983). Sensitive method for the rapid determination of phyate in cereals and products. J. Sci. Food Agric, 34, 14232-14261

8. Hsu, H., Vavak, I., Satterlee \& Miller, G. (1977). A multienzyme technique for estimating protein digestibility. Journal Food Science and Technology, 42(5), 1269-1273

9. ISO 9648; (1988). Determinación del contenido de taninos en sorgo. International Organization of Standardizations, ISO/DIS 9648, 175-215.

10. Fernández Mosquera M (2010). Semilla de chía. Disponible en: http://www.semillasdechia.com/propiedades.html

11. Martínez-Manrique, E., Jiménez, K., Caballero R., Moreno, E. (2005). Influencia del deterioro del frijol (Phaseolus vulgaris) durante su almacenamiento sobre su calidad nutrimental. Memorias del XL Congreso Mexicano de Química. 25 al 29 de septiembre, Morelia, Michoacán, México.

12. Rama, M., Tara, R., Krishnan, C., (1974). Colorimetric estimation of tryptophan content of pulses. Journal Food Science and Technology. 11, 213-216

13. Silva S. A. (2015). Evaluación técnica comercial del aprovechamiento de la semilla de chía (Salvia hispánica) para la elaboración de productos alternos. Facultad de Ingeniería y Ciencias Agropecuarias. Universidad de las Américas.

14. Urbina Manuel (1987). La chía y sus aplicaciones. Chapingo. México. Disponible en: https://chapingo.mx/revistas/revistas/articulos/doc/rga-1826.pdf 